Studium 3 (2008) 226-242

\title{
L’Ecole Sucrière Belge de Glons
}

\author{
HENDRIK DEELSTRA* ET MICHEL PETERS**
}

Curieusement l'Ecole Sucrière Belge de Glons et ses prolongements se sont à première vue perdus dans l'oubli du temps. En 1917 les troupes Allemandes détruisirent l'Ecole et toutes les archives. Cependant les habitants de Glons se souviennent encore de l'existence de l'Ecole, puisque jusqu'en 1917 plusieurs habitations servaient de logements pour de nombreux étudiants venant de Belgique et de l'étranger. ${ }^{1}$ Cet article veut d'abord situer la création de cette Ecole, en 1889, par l'initiative privée de E. Nihoul (1857-1894)², dans le contexte des débats qui eurent lieu à cette époque entre les fabricants de sucre sur la formation professionnelle des ingénieurs spécialistes en matière de fabrication du sucre. François Sachs (1849-1919) 3 et avant lui Victor Beauduin (1845-1904) ${ }^{4}$ exigeaient une formation basée sur un niveau scientifique élevé, tandis que E. Nihoul, en 1889 ingénieur dans

* Hendrik Deelstra, Universiteit Antwerpen (UA), Campus Drie Eiken, Departement Farmaceutisch Wetenschappen, Universiteitsplein 1, 2610 Wilrijk, België E-mail: hendrik.deelstra@ua.ac.be

** Michel Péters, 9 Avenue Henri Monjoie, 4300 Waremme, Belgique. E-mail: mpeters@paque.eiffage.be

1 Sur le site « Glons mon village ma vallée » on apprend que l'école sucrière de Belgique naquit à Glons en 1889, suite à la demande de la Société Générale des Fabricants du Sucre de Belgique, présidée par V. Beauduin, directeur de la Raffinerie Tirlemontoise. En 1892 l'école fut agréée par l'Etat et prit plus tard le nom « d'Institut Polytechnique de Glons ». Les premiers ingénieurs techniciens furent formés en 1893. De nombreuses habitations de Glons servaient à l'époque de logements pour les étudiants venant de Belgique et de l'étranger. En 1917 les troupes allemandes occupèrent l'Institut et détruisirent les archives (http://portail.bricabrac.be/ gotoframe.php3?id=603F).

2 Eugène Joseph Nihoul (1857-1894) naît à Boneffe le 26 octobre 1857. Son père Louis Joseph Nihoul était pharmacien et sa mère Eléonore Josèphe Docq ménagère. Il épousa Marie Anne Valérie Dothée de Glons. Il résidait à Glons en 1881 (rue Brouck Labay). Il décéda en sa maison rue Colombier à Glons le 6 mai 1894. E. Nihoul est inhumé au vieux cimetière de Glons. Eugène Nihoul obtenait le diplôme de pharmacien à l'Université de Liège en 1879. Lors du sixième congrès international pharmaceutique à Bruxelles en 1885, Eugène Nihoul envoya une communication sur la falsification du beurre, il représentait à cette époque le comité agricole du Pays de Herve. Eugène Nihoul fit son entrée en tant que membre de l'Association Belge des Chimistes le 20 décembre 1889 , avec comme référence, le titre d'ingénieur à la sucrerie de Les Waleffes.

3 François Sachs (1849-1919) était sorti en 1871 comme ingénieur de l'Ecole des Arts et Manufactures de l'Université de Gand. Il fut le 14 avril 1887 parmi les fondateurs de l'Association belge des chimistes et devenait le premier secrétaire général de 1887 à 1895 (voir sur ce sujet H. Deelstra et R. Fuks 'La réorganisation fondamentale de l'Association Belge des Chimistes (1898)', Chimie Nouvelle 16 (1989) 1971-1977). Le premier Congrès Internationale de Chimie Appliquée en 1894 à Bruxelles-Anvers était une des ses grandes oeuvres (voir Chimie Nouvelle 13 (1995) 1443-1447). Sur une suggestion de F. Sachs une nouvelle société fut fondée le 14 février 1896 avec comme titre "Société Technique et Chimique de sucrerie de Belgique ». F. Sachs fut le premier secrétaire général, fonction qu'il occupa jusqu’à sa mort (La Sucrerie Belge XXIV (1896) 276-301). Le 15 avril 1889 il était chargé de fonction de rédacteur de La Sucrerie Belge. Il occupa cette fonction pendant vingt-cinq ans. Il organisait aussi le Premier Congrès International de Sucrerie et de distillerie à Liège (5-10 juillet 1905) (La Sucrerie Belge 42 (1914) 386-394).

4 Victor Beauduin (1845-1904) fit ses études à l'Université de Louvain, où il obtint en 1865 le diplôme de docteur en droit. Après un stage à la sucrerie de Rosoux-Goyer, dirigée par son frère Jules, il entra en 1875 à la sucrerie raffinerie Vinckenbosch et Cie qui allait devenir la Raffinerie Tirlemontoise. En 1880 il fut nommé secrétaire de la Société Générale des Fabricants de Sucre et en même temps, directeur du journal La Sucrerie Belge. En 1890 il donna sa démission en tant que directeur du journal. Il se consacra à sa tâche de fabricant de sucre. A partir de 1900 il fut membre du Parlement, et également bourgmestre de Tirlemont (1892-1904).Voir Y. Stinglhamber, 'Beauduin, Victor-François-Joseph', Biographie Nationale 39 (1976) 105-107. Il fut membre honoraire fondateur de l'Association Belge de Chimistes (Bulletin de l'Association Belge des Chimistes 1 (1887) 22) et membre de la Société Technique et Chimique de Sucrerie de Belgique (La Sucrerie Belge 28 (1900) 401). 


\section{L'Ecole Sucrière Belge de Glons}

une sucrerie, voyait la nécessité impérieuse de former directement des collaborateurs capables d'aider efficacement les fabricants de sucre par une formation strictement utilitaire.

Les premiers résultats des recherches sur l'histoire de l'Ecole entre 1889 et 1914, montrent qu'après un début lent l'Ecole Sucrière Belge acquit un rayonnement international comme peut en témoigner l'analyse des cours et programmes, des interrogations et examens, ainsi que des diplômés et de leur situation dans l'industrie du monde entier.

\section{Introduction}

Lors de l'Assemblée Générale de l'Association Belge des Chimistes, le 3 mai 1893, François Sachs (1849-1919) secrétaire général de l'Association prononça un discours ayant pour titre "Ecole de sucrerie et Station Expérimentale ».5 Il y soulignait tout d'abord que les industries «modernes » s'étaient développées exclusivement par des recherches scientifiques nombreuses et patientes. Il fit remarquer que grâce à une pléiade de chercheurs dans le domaine de la chimie, de la physique, de la mécanique, de l'agriculture et d'autres branches de la science, la fabrication du sucre de betteraves connaissait un développement prodigieux. ${ }^{6}$ En 1884, face à la concurrence acharnée, seuls les fabricants disposant des employés capables et bien préparés étaient en mesure de subsister. ${ }^{7}$ Selon les mots de François Sachs la situation en 1893 était semblable.

L'obstacle à un progrès constant était l'absence d'une école spéciale consacrée exclusivement à l'étude de la fabrication du sucre. Selon F. Sachs cette école devait être associé d'office à une Station Expérimentale de fabrication du sucre. ${ }^{8}$ Il expliquait que cette Station serait avantageuse: d'un côté parce que les fabricants pourraient y faire exécuter toutes sortes de recherches quant à l'amélioration de la production, d'autre part parce que les élèves capables auraient l'occasion de faire apprécier leurs connaissances.

Après cette introduction F. Sachs donna un aperçu de la formation sucrière en Europe. Il commença d'abord par l'Allemagne où l'on avait compris la nécessité de l'existence de ces écoles sucrières. L'Allemagne comptait trois écoles de sucrerie et deux stations expérimentales. La plus ancienne et la plus connue de ces écoles de sucrerie était celle de Brunswick fondée en 1876 pas le dr. R. Frühling et par le dr. J. Schulz. ${ }^{9}$ Une deuxième école fut créée,

5 F. Sachs, 'Ecole de Sucrerie et Station Expérimentale', Bulletin de l'Association Belge des Chimistes 7 (1893) 20-25. 6 F. Sachs comparait les chiffres de la production de sucre en Belgique avec les chiffres de l'Allemagne. En Belgique il y avait en 1872, 90 millions de kg et en Allemagne 263 millions de kg; pour 1892 ces chiffres étaient respectivement de 160 millions de kg pour la Belgique et 1225 millions de kg pour l'Allemagne.

7 L'augmentation de la production de sucre en 1884 avait laissé de nombreuses victimes parmi les fabricants de sucre: plus de 40 fabriques avait du payer de leur existence le manque de prévoyance (au 1 octobre 1880 la Belgique comptait 146 fabriques de sucre, La Sucrerie Belge 9 (1880) 73-76).

8 F. Sachs se référait à l'existence des écoles de brasseries. Voir sur ce sujet E. Van Schoonenberghe, 'The Belgian brewing schools', Cerevisia 20 (1995) 32-35 et 'Het ontstaan van het hoger brouwerij-onderwijs in België aan het einde van de $19^{\text {de }}$ eeuw', Cerevisia and Biotechnology 17 (1992) 39-53.

9 Des données sur le fonctionnement de l'école de Brunswick se retrouvent dans différents numéros de La Sucrerie Belge. La ligne directrice de ce programme d'enseignement nous y est donné (La Sucrerie Belge 5 (1877) 206 et 9 (1881) 243-245). L'institut admettait des jeunes gens qui appartenaient déjà à l'industrie sucrière ainsi que ceux qui avaient une vocation. La durée de l'enseignement était de quatre mois (100 jours à 48 heures par semaine), quatre mois consacrés aux études théoriques et surtout aux travaux pratiques en laboratoire. L'enseignement se donnait jusqu'à l'ouverture de la campagne sucrière. Le programme détaillé par semaine est donné dans La Sucrerie Belge 9 (1881) 245. Le corps enseignant (La Sucrerie Belge 18 (1888) 248) avait comme spécialiste pour la fabrication du sucre le dr. Charles Stammer (1882-1893) (voir la nécrologie dans le Bulletin de l'Association Belge de Chimistes 7 (1893) 130). En 1876 le nombre total des élèves de l'Institut depuis la fondation était de 388 (La Sucrerie Belge 12 (1884) 506). Les élèves étaient de différentes nationalités, les résumés $\mathrm{du} 13^{\text {ième }}$ et $14^{\text {ième }}$ rapports respectivement de 1888 et 1889 , nous montrent qu'il y avaient "plusieurs hollandais ", 6 en 1888 et 14 en 1889 et seulement 1 belge en 1889 (La Sucrerie Belge 17 (1889) 185 et 248). 


\section{Hendrik Deelstra et Michel Peters}

annexée à l'école polytechnique de Brunswick. Seuls les élèves ayant terminés les trois années de cours à l'école polytechnique étaient admis dans cette école spécialisée. ${ }^{10}$ Une troisième école de sucrerie, qui avait annexée une Station Expérimentale, était établie à Berlin, ${ }^{11}$ tandis qu'une autre Station Expérimentale était installée à Bernsburg sous la direction de M. Hellriegel. A Vienne il existait une Station Expérimentale dirigée par F. Strohmer. La Russie possédait une école de sucrerie à Smiéla et un laboratoire central à Kieff.

Poursuivant son discours, F. Sachs développa l'évolution de la situation en France. ${ }^{12}$ Après avoir créé des cours spécifiques aux écoles de Physique et de Chimie à Paris, le Gouvernement créa une Ecole Nationale des Industries Agricoles à Douai. Malheureusement l'admission à cette école était exclusivement réservée aux Français.

F. Sachs continua en signalant que pour la Belgique il fallait constater qu'il n'y existait pas d'enseignement spécifique pour les industries « les plus importantes du pays». Il ne comprenait pas pour quelles raisons on n'avait pas encore résolu ce problème. Et pourtant on y avait déjà songé, rappelant qu'en 1881 Victor Beauduin (1845-1904), rédacteur en chef de La Sucrerie Belge avait déjà préconisé la création d'une école de sucrerie.

\section{Prélude}

En effet dans La Sucrerie Belge du $1^{\mathrm{er}}$ février 1881, Victor Beauduin, avait attiré l'attention, dans sa chronique, sur la nécessité d'une formation d'ingénieurs spécialistes dans une école de sucrerie. ${ }^{13}$ Selon V. Beauduin la fabrication du sucre ainsi que la culture de la betterave exigeaient une approche scientifique. En analysant la situation de la fabrication du sucre en Belgique, V. Beauduin arrivait à la conclusion que l'on travaillait plutôt empiriquement et à l'aveuglette. Certes d'année en année les progrès étaient en évolution constante mais ils étaient plus conséquents dans les autres pays ce qui faisait que l'industrie sucrière belge restait en marge des avancées les plus importantes. Il était donc indispensable de monter des laboratoires chimiques dans chaque industrie pour améliorer la production. Mais pour recueillir des bénéfices on avait besoin de chimistes bien formés. Concernant les connaissances des ingénieurs diplômés en Belgique V. Beauduin constatait que ces diplômés ne savaient rien des applications industrielles. Il disait: « les études, quoique nommées spéciales, visent toutes les industries [...] et finalement les ingénieurs préparés à tout, ne connaissent rien! ». On avait donc besoin d'une solution urgente.

Pour trouver une solution V. Beauduin s'orientait vers l'Allemagne avec comme exemple l'Institut de Brunswick où l'on avait trouvé un lien entre les études théoriques et leur application industrielle à la sucrerie. V. Beauduin proposait d'utiliser et d'imiter cette école. Pour l'utiliser il proposait d'un côté de demander à l'Allemagne les hommes dont la Belgique avait besoin pour ces laboratoires et de l'autre côté d'envoyer à cette école des jeunes gens qui connaissaient assez d'allemand pour pouvoir suivre les cours et se perfectionner.

10 Le programme de cette spécialité ne comportait que quatre cours: méthodes spéciales de la fabrication du sucre ( $4 \mathrm{~h}$ par semaine), culture de la betterave ( $2 \mathrm{~h}$ par semaine), chimie agricole ( $2 \mathrm{~h}$ par semaine) et chimie pratique (toute la journée) (voir note 5).

11 L'ancien directeur de l'école sucrière de Berlin était le dr. Ch. Scheibler (1827-1899), remplacé en 1883 par le dr. A. Herzfeld ( 1854-1928).

12 On trouve des informations sur l'Ecole Sucrière ouverte à Douai (France) en 1893 dans le rapport du jury international par L. Dabat, classe 5: enseignement spécial agricole (Rapports du jury international de l'Exposition Universelle Internationale de 1900 à Paris, imprimerie nationale (1904) 290-304) et dans un article de V. Beauduin dans La Sucrerie Belge 12 (1884) 118-119.

13 V. Beauduin, 'Chronique', La Sucrerie Belge 9 (1881) 179-183. 


\section{L'Ecole Sucrière Belge de Glons}

Ensuite, il proposait également d'imiter les Allemands. Puisque ni le Gouvernement, ni les écoles spéciales n'agissaient spontanément pour résoudre ce problème il demandait que l'Association Belge des Fabricants prenne l'initiative. Pour être complète et donner des fruits, V. Beauduin proposait d'associer à la création d'une Ecole sucrière, une Station Sucrière, semblable aux stations agricoles. ${ }^{14}$

Suite a cette chronique il y eut deux sortes de réactions. D'un côté deux lettres furent envoyées de la part de Julien De Puydt ${ }^{15}$ à La Sucrerie Belge. ${ }^{16}$ Dans la première datée du 4 février 1881, l'auteur se réjouissait de l'intérêt porté au sujet développé dans la chronique du 1er mai 1881, et avec lequel il était entièrement d'accord tant sur l'importance que sur l'urgence. J. De Puydt ne se disait par contre pas convaincu de pouvoir résoudre le problème du manque et de l'absence de chimistes par l'envoi de belges en Allemagne pour y faire un stage industriel, car très peu d'ingénieurs chimistes maîtrisaient assez d'allemand pour profiter des leçons données. Même chose quant à l'appel en Belgique de chimistes allemands, ceux-ci ne connaissant pas assez de français ou de flamand pour se faire comprendre et acquérir l'autorité nécessaire. A la fin de cette lettre il ajoutait que ces professeurs «ne seraient probablement pas la fleur de l'école allemande. » Non, pour J. De Puydt il y avait en Belgique assez d'écoles où l'on enseignait sérieusement la chimie et la chimie industrielle, mais, par contre, il manquait de connaissances pratiques. De plus il y avait, ce qu'appelait J De Puydt, entre les diplômés et les patrons des problèmes " de continuité ». Pour éviter la méfiance entre les patrons et les ingénieurs belges, il proposait de créer une "sucrerie-école ». L’Association Sucrière de Belgique pouvant, d'après lui, acquérir une vieille sucrerie mise à vendre. Cette école aurait un directeur et des professeurs peu nombreux. Les produits de l'usine serviraient à couvrir les frais et à amortir le capital. Il lui semblait que les ingénieurs sortis de pareille école, fondée par les sucriers eux-mêmes, inspireraient beaucoup plus de confiance.

Dans sa seconde lettre datée du 25 février 1881 J. De Puydt s'expliquait longuement sur son projet qu'il appelait alors « l'usine-école ». Il décrivait en détail le fonctionnement que pourrait avoir cette usine-école où les sessions devraient commencer au mois d'août avec des cours théoriques et des excursions sur le terrain. Ensuite, au début du mois d'octobre s'ouvrirait «la fabrique-école », dans laquelle les professeurs et les élèves prendraient les rôles de directeurs et d'ouvriers. Pendant les quatre mois de fonctionnement de l'usine, les patrons et les futurs ingénieurs spécialistes seraient en mesure de s'apprécier

14 Le 25 septembre 1871 une association pour la fondation de stations agricoles en Belgique fut fondée. Grâce aux subsides obtenus du gouvernement l'association crée la première station agricole à Gembloux. En 1872 Arthur Petermann (1845-1902) en devenait le premier directeur. Une seconde station fut fondée en 1875 à Gand et en 1878 deux nouveaux laboratoires agricoles virent le jour à Liège et à Hasselt. En 1883, les quatre stations furent reprises par l'Etat. En 1884 trois nouveaux laboratoires de l'Etat furent fondés: Anvers, Louvain et Mons. Enfin, en 1887 un arrêté royal subdivisa la Station de Gembloux en deux entités distinctes: une station agronomique et le laboratoire agricole. A partir de 1890 la station agronomique de Gembloux devint un établissement de recherche et les sept autres laboratoires d'analyses de l'Etat furent mis à la disposition du public pour faire exécuter l'analyse des produits agricoles (voir Station agronomique et laboratoires d'analyses de l'Etat, 1871-1896, rapport par Arthur Petermann, Bruxelles, 1896).

15 Julien De Puydt (1842-1919) était ingénieur mécanicien sorti en 1864 de l'Ecole des Mines de Mons (voir Association des Ingénieurs de l'Ecole des Mines à Mons (1839-1909)). En 1864 il fut directeur de la Sucrerie d'Attre à Mons, de 1869-1874 fut ingénieur directeur de la sucrerie du Pont Canal près de Mons, à partir de 1874, ingénieur chimiste à Bruxelles et à partir de 1878, ingénieur chimiste à Anvers. Il fut membre fondateur de l'Association Belge des Chimistes. (Bulletin de l'Association Belge des Chimistes 1 (1887) 22) et membre fondateur de la Société Technique et Chimique de Sucrerie en Belgique (La Sucrerie Belge 25 (1896) 381).

16 J. De Puydt, 'Correspondance', 4 février 1881, La Sucrerie Belge 9 (1881) 211-212. et J.De Puydt, 'Correspondance', 25 février 1881, La Sucrerie Belge 9(1881) 252-257. 


\section{Hendrik Deelstra et Michel Peters}

mutuellement. De même pendant ce temps de formation l'usine-école pourrait être un vaste laboratoire d'essai. Enfin, pour la faisabilité sur le plan financier J. De Puydt soumettait plusieurs propositions.

Dans sa chronique du 15 février $1881 \mathrm{~V}$. Beauduin donna un résumé de ces propositions du 1 février 1881 et se réjouissait de la lettre de J. De Puydt, mais il ajoutait qu'il trouvait difficile de réaliser ce projet d'usine-école en Belgique. V. Beauduin préférait la création d'application industrielle sucrière dans une des écoles spéciales existantes. Dans sa chronique du 15 mars 1881, V. Beauduin toujours, répondait à la deuxième lettre détaillée de J. De Puydt. Il avait une attitude beaucoup plus positive face aux propositions de J. De Puydt quant à son idée sur la création d'une sucrerie-école, qui constituerait en effet l'alliance étroite de l'école et de l'atelier, de la théorie et la pratique dont la nécessité s'imposait pour l'industrie sucrière. Il soulignait seulement que la réalisation des idées de J. De Puydt exigerait un effort commun assez énergique. Il terminait en disant que «les généreux projets d'une homme estimé de tous méritent la reconnaissance de l'industrie ». ${ }^{17}$

D’un autre côté, V. Beauduin, dans sa chronique du 15 mars 1881 se plaignait « des accusations aussi injuste que malveillantes dont il avait été l'objet ». Ces accusations avaient été publiées dans le Journal agricole du Brabant-Hainaut du 20 février 1881. Ce journal accusait V. Beauduin, d'abord, de s'être montré peu aimable envers les fabricants en leur donnant un brevet d'incapacité, le journal déclarait ensuite que le corps professoral des Ecoles Spéciales était blessé dans son honneur professionnel. V. Beauduin réfuta ces deux attaques d'une façon très claire en soulignant que tout d'abord les fabricants avaient, à une réunion du 2 mars, eu l'occasion de s'opposer à cette critique, mais qu'au contraire les fabricants y avaient témoigné toute leur sympathie. Ensuite V. Beauduin souligna que nul reproche n'avait été adressé aux professeurs et fit la remarque qu'il s'était seulement borné à signaler le manque de connaissances pratiques, c'est-à-dire l'insuffisance d'organisation des écoles. A côté de cette polémique, un correspondant anonyme, ingénieur agricole, écrivit au même journal que le Directeur de La Sucrerie Belge était trop avocat pour pouvoir porter un jugement sur une question d'enseignement industriel: il trouvait inadmissible "les attaques immodérées contre un établissement où il avait puisé toutes ses connaissances $»{ }^{18}$ L'auteur lui ferait injure en lui proposant un voyage en Allemagne, voyage qu'il appela «l'engouement de Victor Beauduin ». En réponse à cet diatribe anonyme, V. Beauduin cita les chiffres de la production de sucre des années 1871 à 1881 en Belgique, en France et en Allemagne. ${ }^{19} \mathrm{~A}$ l'aide de ces chiffres V. Beauduin montrait qu'en 1881 le grand maître de l'industrie sucrière était l'Allemagne. ${ }^{20}$

17 V. Beauduin, 'Chronique', La Sucrerie Belge 9 (1881) 203-205 et V. Beauduin, 'Chronique', La Sucrerie Belge 9 (1881) 240-243.

18 Il s'agissait de l'Institut Agronomique de Gembloux fondé en 1860. Une ferme fut annexée à l'Institut pour l'instruction pratique des étudiants. La durée des études était de trois ans, le diplôme obtenu était celui d'ingénieur agricole. En 1878 Justin Piret, qui avait succédé en 1874 au professeur Phocas Lejeune, premier directeur de l'Institut et titulaire du cours d'économie rurale, publiait un livre intitulé Etude sur le capital agricole. A la page 61 on pouvait y lire « On dit et répète de plus en plus que l'agriculture doit devenir industrielle $[. .$.$] il est temps que le cultivateur ne se laisse plus guider par les prescriptions d'une routine aveugle$ $[\ldots]$ en agriculture et en industrie, les grands progrès sont dus à la chimie, [...] qui ne sont plus des sciences purement spéculatives mais des sciences appliquées ».

19 Production du sucre (en tonnes) de 1871-1881 en Belgique, France et Allemagne

Belgique France Allemagne

Moyenne (1871-1875) $\quad 72.700 \quad 410.000 \quad 266.000$

$\begin{array}{llll}\text { Moyenne (1876-1881) } & 60.000 & 334.000 & 405.000\end{array}$

(Voir La Sucrerie Belge 9 (1881) 243) 


\section{L'Ecole Sucrière Belge de Glons}

L'engagement de chimistes dans les sucreries n'était pas chose courante pour l'époque. Beaucoup de directeurs de sucrerie cherchaient, en effet, à éviter les coûts de contrats de longue durée en ne s'attachant un ingénieur chimiste que pour la durée de la campagne. Constant Tydgadt, le successeur de V. Beauduin, comme rédacteur de La Sucrerie Belge se fit l'écho de ce problème dans la revue La Sucrerie Belge en 1882-1883:

Nous avons eu l'occasion à maintes reprises d'entretenir nos lecteurs de la pénurie de bons chimistes de sucrerie à même de faire la surveillance chimique de la fabrication. La même disette se fait sentir cette année et nous connaissons plusieurs fabricants qui, malgré des recherches actives, n'ont pu trouver pour leurs usines ces auxiliaires si nécessaire aujourd'hui dans les fabriques.

Cet état de choses est évidemment préjudiciable et fâcheux; qu'il nous soit permis d'ajouter que les fabricants en sont eux-mêmes quelque peu responsables.

En général toutes les carrières en Belgique ne sont pas dépourvues d'aspirants, bien au contraire. S'il ne s'en trouve pas pour celle d'ingénieur chimiste de sucrerie en nombre voulu, c'est que les propriétaires de fabriques n'ont pas encore réglé les conditions de cette position de façon à en faire une carrière convenable quoique provisoire. Provisoire elle peut l'être, en ce sens que les jeunes gens de valeur peuvent ambitionner mieux au bout de quelques années. Convenable elle doit l'être, en ce sens qu'il n'est point possible de restreindre l'engagement de ces chimistes à la durée de la fabrication pour les abandonner ensuite.

Une industrie ne saurait de cette façon ni s'attacher son personnel, ni le former d'une manière sérieuse. Ce n'est guère qu'au bout d'une ou deux campagnes que le chimiste connaîtra assez la fabrication, le personnel et l'usine pour rendre tous les services que l'on peut en attendre.

Le lâcher après la fabrication terminée, c'est presque toujours s'exposer à ne plus le revoir dans l'industrie sucrière. Sans doute la besogne fait un peu défaut en été, mais [...] le sacrifice qui peut en résulter pour l'industriel sera largement récompensé pendant la fabrication suivante. ${ }^{21}$

Cette position explique certainement le processus qui amena tous les acteurs de cette industrie à envisager sérieusement la formation de chimistes et d'ingénieurs chimistes de sucrerie quelques années plus tard.

Le 1 juin 1891 on pouvait lire dans La Sucrerie Belge que le conseil d'administration de la Société Générale des Fabricants de Sucre était saisi d'un projet tendant à la création d'une école sucrière en Belgique. ${ }^{22} \mathrm{C}$. Tydgadt, soulignait que cette école devrait être scientifique. Pour aborder les études de cette école les élèves devraient être en possession d'un diplôme de l'enseignement supérieur. Cependant cette condition d'admission ne serait pas nécessaire pour quelques élèves libres, qui pourraient suivre certains cours. Le Journal des Fabricants de Sucre du 8 juillet 1891 rapporta également que la Société Générale

20 La polémique entre La Sucrerie Belge et le Journal de la Société Agricole du Brabant-Hainaut continuait encore dans le Journal Agricole, puis par une réplique de La Sucrerie Belge le 15 avril 1881 (La Sucrerie Belge 9 (1881) 285286), pour se terminer par un bref article le 8 mai 1881, dans le Journal Agricole (cité par La Sucrerie Belge 9 (1881) 341).

21 C. Tydgatdt, 'Ecole de Sucrerie de Belgique', La Sucrerie Belge 19 (1891) 365-267.

22 Journal des Fabricants de sucre, 8 juillet 1891. 


\section{Hendrik Deelstra et Michel Peters}

des Fabricants au Sucre de Belgique avait mis à l'étude un projet de création d'une école sucrière.

\section{L'Ecole Sucrière Belge de Glons}

Dans son discours devant l'Assemblée Générale de l'Association Belge des Chimistes le 3 mai 1893, F. Sachs, ne mentionnait que brièvement l'existence de l'école de sucrerie de Glons. Il disait que l'initiative privée avait essayé d'établir cette école en ajoutant que cet établissement ne pouvait " évidemment " pas être la solution complète du problème. Selon lui, il fallait quand même, rendre un juste hommage au courage de ses auteurs.

On peut supposer que plus tard F. Sachs revint sur sa position. Manifestement la Société Générale des Fabricants de Sucre n'avaient pas pu créer une école sucrière sur des bases scientifiques solides comme à l'Institut Agronomique de Gembloux. En effet F. Sachs envoyait son fils Henri ${ }^{23}$ à l'Ecole Sucrière Belge de Glons, où celui-ci obtenait son diplôme en 1908. Dans quelques registres de " procès-verbaux des examens de sortie pour les années 1905-1909» on trouve parmi les membres du jury final le nom de F. Sachs pour le 10 août 1908 et le 9 août $1909 .{ }^{24}$

A l'occasion du 25ième anniversaire de l'Institut Polytechnique, anciennement dénommé Ecole Sucrière Belge de Glons, le 12 juillet 1914 le directeur Paul le Bussy (1867-1950) ${ }^{25}$ témoignait de l'origine de l'Ecole Sucrière. ${ }^{26}$ Eugène Nihoul (1857-1894), pharmacien de formation avait tourné son activité vers la fabrication du sucre, comme le disait P. le Bussy:

Esprit sagace, chimiste averti, sucrier consommé, [Nihoul] eut tôt fait de discerner les imperfections et les vices des méthodes adoptées pour le contrôle du travail, il eut tôt fait de reconnaître les dangers d'une conduite empirique de la fabrication et les bénéfices qui devait donner un travail scientifiquement conduit et surveillé. Il eut tôt fait de se rendre compte que le fabricant de sucre d'alors, trop souvent industriel quelconque, et sans préparation spéciale suffisante, avait un besoin impérieux, de collaborateurs capables de l'aider efficacement. De là à la pensée de former ces collaborateurs il n’y avait qu'un pas. Il fut vite franchi et en mars 1889 Eugène Nihoul fondait l'Ecole Sucrière Belge à Landen, dans une modeste maison avec comme devise: «Travail Sciences Progrès » ${ }^{27}$.

23 Henri Sachs, fils de François Sachs, né à Gembloux le 28 février 1888, diplômé en 1908 de l'Ecole Sucrière Belge (voir La Vallée du Geer, du 27 août 1908), devenait immédiatement membre de la Société Technique et Chimique de Sucrerie de Belgique (voir la liste des membres, La Sucrerie Belge 39 (1911) 252). Suite à une initiative de Lucien Beauduin, président, la Société Technique et Chimique de Belgique avait voulu contribuer à la célébration du Centenaire de l'Indépendance Nationale en instituant un concours sur l'Historique de l'Industrie Sucrière en Belgique. A l'Assemblée Générale du 27 mai 1931, Henri Sachs fut proclamé premier lauréat de ce concours (voir La Sucrerie Belge 50 (1931) 13-16). Henri Sachs fut nommé Secrétaire Général adjoint du IV ${ }^{e}$ Congrès International Technique et Chimique des Industries Agricoles à Bruxelles en 1935 (ce congrès fut un prolongement du congrès de 1905, voir note 3 ).

24 Ces registres se trouvent dans les archives de la Haute Ecole Rennequin Sualem, 6 Quai Gloesener à Liège.

25 Paul le Bussy (1867-1950) est appelé jusque 1925, Bussy. Par jugement du 18 février 1925, une rectification patronymique change le nom Bussy en le Bussy. Paul (le) Bussy est le fils de Théophile Bussy (1838-1912) et de Melanie Malherbe. Il est le petit fils de Arnold Paul Bussy (1797-1865) Bourgmestre de Flémalle Grande. Paul est né à Herstal le 21 juillet 1867, il épousa à Flône le 9 mai 1895, Marie Christine Hock. Il décéda à Liège le 30 juin 1950 à l'âge respectable de 82 ans..Il était membre de la Société Technique et Chimique de Sucrerie en Belgique (voir Liste des membres 1914-1915', La Sucrerie Belge 42 (1914) 543).

26 'XXVe Anniversaire de l'Institut polytechnique de Glons', La Sucrerie Belge 38 (1914) 504 et 554.

27 Paul Bussy, 'Discours donné lors du 25eme anniversaire de la fondation de l'Ecole Sucrière Belge', Supplément au journal La Vallée du Geer $n^{\circ} 30$ du 23 juillet 1914. 


\section{L'Ecole Sucrière Belge de Glons}

Nombre total des diplômés de L'ecole Sucrière Belge.

\begin{tabular}{|c|c|c|c|c|c|c|c|c|c|c|}
\hline \multirow{2}{*}{$\begin{array}{l}\text { Grades } \\
\text { Avec la plus grande } \\
\text { distinction }\end{array}$} & \multicolumn{2}{|c|}{1904} & \multicolumn{2}{|c|}{1905} & & \multirow[t]{2}{*}{1906} & \multicolumn{2}{|c|}{1908} & \multicolumn{2}{|c|}{1910} \\
\hline & & & 1 & 1 Polonais & & & 1 & 1 Polonais & & \\
\hline Avec grande distinction & 4 & $\begin{array}{l}1 \text { Polonais } \\
1 \text { Italien } \\
1 \text { Français } \\
1 \text { Belge }\end{array}$ & 1 & 1 Russe & 5 & $\begin{array}{l}2 \text { Italiens } \\
1 \text { Serbe } \\
1 \text { Russe } \\
1 \text { Péruvien }\end{array}$ & 4 & $\begin{array}{l}3 \text { Polonais } \\
1 \text { Russe }\end{array}$ & 2 & 2 Polonais \\
\hline Avec distinction & 8 & $\begin{array}{l}3 \text { Italiens } \\
2 \text { Espagnols } \\
2 \text { Français } \\
1 \text { Russe }\end{array}$ & 4 & $\begin{array}{l}2 \text { Espagnols } \\
1 \text { Russe } \\
1 \text { Polonais }\end{array}$ & 2 & $\begin{array}{l}1 \text { Espagnol } \\
1 \text { Italien }\end{array}$ & 10 & $\begin{array}{l}5 \text { Italiens } \\
2 \text { Français } \\
1 \text { Polonais } \\
1 \text { Espagnol } \\
1 \text { Brésilien }\end{array}$ & 4 & $\begin{array}{l}2 \text { Polonais } \\
1 \text { Espagnol } \\
1 \text { Belge }\end{array}$ \\
\hline De manière satisfaisante & 4 & $\begin{array}{l}1 \text { Russe } \\
1 \text { Polonais } \\
1 \text { Espagnol } \\
1 \text { Italien }\end{array}$ & 11 & $\begin{array}{l}4 \text { Brésiliens } \\
3 \text { Polonais } \\
1 \text { Russe } \\
1 \text { Espagnol } \\
1 \text { Français } \\
1 \text { Américain }\end{array}$ & 4 & $\begin{array}{l}2 \text { Italiens } \\
1 \text { Espagnol } \\
1 \text { Belge }\end{array}$ & 12 & $\begin{array}{l}5 \text { Italiens } \\
3 \text { Espagnols } \\
2 \text { Belges } \\
1 \text { Polonais } \\
1 \text { Français }\end{array}$ & 9 & $\begin{array}{l}4 \text { Italiens } \\
2 \text { Polonais } \\
1 \text { Belge } \\
1 \text { Espagnol } \\
1 \text { Américain }\end{array}$ \\
\hline Total & 16 & & 17 & & 11 & & 26 & & 15 & \\
\hline
\end{tabular}

Le siège de celle-ci fut transféré à Glons en 1891. En 1893 il transférait son école dans un immeuble spécial à Glons, immeuble qu'il avait constitué en reliant ensemble plusieurs petites maisons. En 1892 l'école fut agréée par l'Etat. Le nombre d'élèves, 7 au début, s'éleva à 12, 18, 25 puis à 32 . La réussite était brillante. Hélas! Eugène Nihoul eut à peine le temps de voir son œuvre prendre son envol. Il mourut en 1894 en pleine force de l'âge. Mais il avait eu le temps de s'entourer de personnes de valeur, comme le soulignait son successeur Paul le Bussy.

Cette école eut un succès certain et bientôt l'espace vint à manquer. Des voix s'élevaient et fustigeaient le pouvoir communal glontois, réclamant des autorités compétentes la construction de locaux plus spacieux pour cette école qui abritait des étudiants de plus de 40 nationalités. Elles furent finalement entendues et, le 2 mai 1907, le Directeur de l'Ecole Sucrière, Paul le Bussy exprimait sa satisfaction: «La prospérité croissante de l'établissement en cette année 1907, rend aujourd'hui réalisable la construction d'une nouvelle école plus spacieuse, non loin de l'école actuelle, sur le terrain qui borde la route $»^{28}$.

L'établissement scolaire se trouvait en pleine mutation. Déjà en 1907, les diplômes furent libellés différemment. On n'y mentionnait plus l'Ecole Sucrière mais bien l'Institut Polytechnique. Il est certain que l'école se déplaça dans un nouveau et vaste bâtiment pour l'année académique 1908-1909. En effet le 10 août 1908, l'Institut Polytechnique accueillait 60 récipiendaires dans ses nouveaux locaux. Il fut même question d'accepter quelques demandes de demoiselles étrangères.

On recensait à cette période comme étudiants étrangers: 3 Autrichiens, 11 Italiens, 3 Espagnols, 1 Sud-Américain, 15 Russes et 25 Polonais. Chose étonnante pour l'époque, dans un petit village! La population de Glons dépassait alors tout juste les 2000 âmes. 


\section{Hendrik Deelstra et Michel Peters}

En 1917 les troupes Allemandes occupèrent l'Institut et détruisirent toutes les archives. L'Institut se déplaça alors à Liège. Paul le Bussy en resta le directeur jusqu'en 1933, lorsque son fils Charles le Bussy le remplaça. En 1953, l'Institut Polytechnique privé passa sous la tutelle de la Ville de Liège, à l'initiative de Maurice Destenay, Bourgmestre. L'Institut de la ville de Liège disposait d'une section biochimie et tannerie qui complétaient ainsi l'éventail des formations.

C'est en 1964 que l'Institut Polytechnique s'installa dans ses locaux actuels, Quai Gloesener à Liège. En 1969, Charles le Bussy céda sa place à la direction de l'école. En 1977, l'Institut Polytechnique fusionna avec deux autres instituts supérieurs: celui de Seraing, créé en 1953, et celui de Waremme, créé en 1956. Il prit le nom d'Institut Supérieur Industriel Liégeois (ISIL), et fut géré de concert par la Ville et la Province de Liège. En 1989, 100 ans après ses débuts l'Ecole Sucrière Belge devenue l'ISIL passa sous la tutelle de la Province de Liège et devint l'Institut Supérieur de la Province de Liège. Elle fait maintenant partie intégrante des Hautes Ecoles Rennequin Sualem.

\section{Organisation et Programmes des Cours}

Une annonce publicitaire parue dans La Sucrerie Belge en 1897 nous apporte, à propos de cette école, quelques informations. ${ }^{29}$ On apprend que l'école, fondée en 1889 était placée sous le patronage du gouvernement, de la province et de la Société générale des Fabricants de sucre de Belgique. ${ }^{30}$ Les cours étaient répartis sur une année entière. Une première section s'ouvrait à Glons en octobre pour se terminer fin février. L'école s'était adjointe à l'Institut Polytechnique de Liège pour donner les cours de la première section. La deuxième section commençait le premier mars pour finir en août. Le diplôme obtenu étant celui d'ingénieur-sucrier.

Le prospectus et le programme des cours de l'Ecole Sucrière Belge Glons de 1901, nous donne: les membres du comité, les membres du corps professoral, le but de l'Ecole, le règlement et ensuite la description détaillée de chaque cours. ${ }^{31}$

L'école était en effet placée sous le patronage de la Société Générale des Fabricants de Sucre de Belgique. Le délégué choisi par cette Société pour la représenter au Comité de l'Ecole était Jules Halbart, fabricant de sucre à Liers. Les autres membres étaient Victor Beauduin, membre de la Chambre des Représentants et Bourgmestre de Tirlemont, Hyacinthe Polet, conseiller provincial à Paifve, Auguste Aulard, fabricant de sucre à Genappe, Lucien Beauduin (1869-1946) ${ }^{32}$, neveu de Victor, fabricant de sucre et raffineur à Tirlemont et Paul le Bussy, le directeur de l'Ecole.

Les membres du corps professoral étaient: Paul Bussy, chimiste-conseil, ingénieur de sucrerie, professeur de chimie et de technologie sucrière ${ }^{33}$; Armand Chabot, ingénieur,

29 'Annonce', La Sucrerie Belge, $26 \mathrm{eme}$ année $\mathrm{n}^{\circ} 1$ du $1^{\mathrm{er}}$ septembre 1897 page (publicitaire) 33.

30 L'Annuaire 1961-1962 de l'Association Royale des Ingénieurs techniciens de l'Institut Polytechnique de la ville de Liège, fondée en 1891, donnait un bref historique de l'Ecole (voir L'annuaire 1961-1962 de l'Association Royale des Ingénieurs Techniciens sortis de l'Institut Polytechnique de la Ville de Liège (Liège 1961) 154. Il est dit que "pour parer au manque des techniciens belges spécialisés dans la sucrerie, la Société générale des fabricants du sucre de Belgique, avec à la tête V. Beauduin, décida de fonder une école des chimistes et d'ingénieurs spécialisés en sucrerie. Cette école ouvrait ces portes en 1889, sous la direction d'une commission de trois membres: MM. E. Nihoul, P. Bussy et M. Winants».

31 Ecole Sucrière Belge (Glons), Prospectus et Programme des cours (Liège 1901) 30.

32 Pour Lucien Beauduin (Henri-Joseph) (1869-1946), voir Y. Stinglhamber, Biographie Nationale 39 (1976) 103-105.

33 Programme des cours: 'Technologie Sucrière: réception des betteraves, extraction des jus, épuration des jus, évaporation, cuite, turbinage, travail des bas produits et autres. Chimie Sucrière: la betterave à sucre, études des sucres, saccharimétrie, analyse de la betterave, analyse de fabrication'. 


\section{L'Ecole Sucrière Belge de Glons}

docteur en sciences naturelles, professeur de chimie générale et de chimie analytique 34 ; Félix Rigaux, ingénieur, professeur du cours des industries de la fermentation ${ }^{35}$; Mathieu Winants, docteur en sciences physiques et mathématiques, professeur de physique, de mécanique rationnelle et le dessin géométrique ${ }^{36}$; Léon Jowa, ingénieur des Arts et Manufactures, professeur de mécanique appliquée et de dessin industriel ${ }^{37}$; Oscar Decostre, ingénieur-mécanicien, ingénieur-électricien, professeur d'électricité ${ }^{38}$; Emile Barlet, avocat, professeur de législation et comptabilité sucrières ${ }^{39}$; Laurent Depaifve, professeur agrégé de l'enseignement moyen, professeur de mathématiques ${ }^{40}$ et Honoré (ou Henri) Duvivier (gendre d'E. Nihoul), chimiste, chef des travaux de laboratoire. ${ }^{41}$

Le but de l'école était de former des gens capables de remplir sans aucune difficulté diverses fonctions dans l'industrie de sucre comme par exemple chimiste, chef de fabrication, comptable et même directeur. Le programme était conçu de manière à supprimer tout ce qui avait un caractère purement spéculatif, en orientant les études vers un but purement pratique et en réduisant leur durée au strict minimum, tout en les maintenant au niveau de l'enseignement supérieur. Dans le programme des cours de l'Institut Polytechnique de Glons-Liège de 1927 tout était résumé dans la phrase suivante: « il constitue donc une école supérieure à programme condensé et débarrassé des branches ou rameaux superflus $» .^{42}$ Les études étaient donc dirigées vers un but strictement utilitaire. ${ }^{43}$

En 1901 le programme s'était étendu aux autres branches importantes de l'industrie, d'abord les industries de fermentation, mais aussi les industries d'engrais, les charbonnages, les haut fourneaux, voir même les usines métallurgiques. En 1901 le corps professionnel comptait 9 professeurs, parmi lesquels P. le Bussy, directeur de l'école et responsable des cours de chimie et de technologie sucrières, Armand Chabot responsable pour la chimie générale et la chimie analytique, Felix Rigoux, responsable des cours des industries de fermentation, Mathieu Winants, le co-fondateur de l'école, responsable des cours de physique, de mécanique rationnelle et de dessin géométrique, et enfin Honoré Duvivier, répétiteur et responsable des travaux de laboratoire.

34 Programme des cours: 'Chimie Générale: notions préliminaires, chimie minérale ou inorganique, chimie organique. Chimie analytique chimie qualitative, chimie quantitative'.

35 Programme des cours: 'Industries de la fermentation: distillerie, vinaigrerie, brasserie, industries diverses'.

36 Programme des cours: 'Mécanique rationnelle: cinématique, statique, dynamique. Dessin géométrique. Eléments de Physique industrielle: attraction, chaleur (thermométrie, changements d'état des corps, transmission de la chaleur, calorimétrie)'.

37 Programme des cours: 'Mécanique appliquée: principaux matériaux, résistance des matériaux, des assemblages que l'on rencontre dans les charpentes métalliques et dans la construction des machines, étude de la machine à vapeur et de son générateur. Dessin industriel: épures, croquis à main levée’.

38 Programme des cours: 'Electricité industrielle: électrostatique, magnétisme, électricité dynamique, circuit électrique, effets des courants, électrolyse, électro-magnétisme, électricité produite par actions mécaniques, éléments de machines à courants alternatifs et polyphasés, moteurs électriques et transports d'énergie électrique, lignes électriques, éclairage électrique, accumulateurs, télégraphie, téléphonie, traction électrique’. 39Programme des cours: 'Législation \& comptabilité sucrières: législation sucrière, comptabilité sucrière'.

40 Programme des cours: 'Mathématiques: arithmétique, géométrie plane, géométrie dans l'espace, algèbre, trigonométrie, arpentage'.

41 Programme des manipulations de laboratoire: 'Travail de verre, chauffage, montage des appareils, pulvérisation, pesées, mesures de liquides, précipitation, et autres opérations de laboratoire. Analyses par voie sèche, par voie humide. Dosage de l'azote, de l'acide phosphorique, de la potasse. Analyse des sels et produits agricoles, des engrais minéraux, végétaux tourteaux divers, et des minerais'.

42 Institut Polytechnique Liège, Programme des Cours (Forest - Bruxelles, 1927) 9.

43 Institut Polytechnique de Glons (Liège) Prospectus, Programme des Cours (Hasselt 1908) 11. 


\section{Hendrik Deelstra et Michel Peters}

Les cours étaient répartis en deux sections ou années d'études. La première section préparatoire comportait les cours des sciences en général. La seconde, section sucrière proprement dite comprenait des cours appliqués à l'industrie du sucre. Pour entrer en seconde section il fallait passer un examen d'entrée qui portait sur les matières de la première section. Les porteurs des diplômes d'ingénieur ou de docteur en sciences étaient dispensés de cet examen. L'école organisait régulièrement des excursions dans les sucreries, raffineries et autres établissements industriels.

En 1906 la durée des études passa de deux à trois ans. L’objectif primordial de l'Institut Polytechnique restait de former des ingénieurs tout d'abord pour l'industrie sucrière, mais aussi pour toutes les autres industries. Les cours étaient répartis en trois sections ou années d'études. Dans le règlement était inscrit qu'un étudiant pouvait être admis à suivre en même temps les cours de deux années d'études. Après des examens passés au moins d'une manière satisfaisante l'étudiant obtenait le diplôme d'ingénieur industriel (N.B. Ce diplôme constituait en même temps un diplôme d'ingénieur spécial en sucrerie). Le programme des cours de 1908 nous apprends qu'il y avaient plusieurs changements par rapport au programme de 1901 de l'Ecole Sucrière Belge, maintenant nommé Institut Polytechnique de Glons. ${ }^{44}$ Le comité voulait élargir le but de l'école, de manière à former des ingénieurs non plus pour la seule industrie sucrière, mais pour l'industrie en général. On avait ajouté plusieurs cours par exemple un cours d'architecture industrielle et un cours d'électricité.

Parmi les membres du Comité, V. Beauduin (décédé en 1904) ne figure plus. Hyacinthe Polet est toujours présent, maintenant comme membre de la Chambre des Représentants. Comme nouveau membre il y avait Julien Delaite, docteur en sciences et conseiller provincial à Liège. Les membres du corps professoral sont au nombre de 8 (cfr. 9 en 1901). Ne paraissent plus: A. Chabot, F. Rigaux, O. Decostre et E. Barlet. Maurice Duguet (Liège), docteur en sciences naturelles et Répétiteur et Chef des travaux de la Chimie général à la Faculté de l'Université de Liège remplaçait Armand Chabot comme responsable de la chimie générale et de la chimie analytique. Un autre nouveau professeur est Edmond Vrancken, ingénieur mécanique et directeur de sucrerie, responsable des cours sur la physique industrielle et d'application de ces cours à la sucrerie, de la résistance des matériaux et de mécanique appliquée et enfin Jean Bruscantini, docteur en sciences physiques et mathématiques donnait les cours de mathématiques supérieures, de mécanique rationnelle, de thermodynamique et hydraulique, et de l'électricité industrielle. Le programme de 1908 énumère 17 personnes citées comme membres des jurys d'examens. ${ }^{45}$

44 Ibidem 42.

45 Les membres des jurys d'examen en 1908 étaient: A. Aulard, ingénieur chimiste; J. Bertrand, docteur en sciences naturelles; P. Beyaert, ingénieur électricien, A. Chabot, docteur en sciences naturelles et ingénieur agricole; O. Decostre, ingénieur mécanicien et ingénieur électricien; J. Delaite, docteur en sciences naturelles; A. Duchesne, ingénieur des Mines et ingénieur électricien, répétiteur à la Faculté technique de l'Université de Liège; C. Fooz, professeur à l'école mécanique de Liège; H. Fraikin, ingénieur brasseur à Roclenge; A. Gouvion, ingénieur des Arts et Manufactures; C. Hock, ingénieur des Mines; E. Légier, ingénieur chimiste, rédacteur en chef de La Sucrerie indigène et coloniale à Paris; V. Mirlant, ingénieur, professeur de chimie analytique à l'école spécial d'industrie et de Mines du Hainaut; H. Pellet, chimiste-conseil des Sucreries de Say et d'Egypte à Paris; H. Phillippot, docteur en sciences physiques et mathématiques; E. Silz, ingénieur chimiste, secrétaire générale de l'Association des Chimistes de sucrerie et de distillerie de France, Paris; et de F. Verbièse, ingénieur chimiste à Lille. 


\section{L'Ecole Sucrière Belge de Glons}

\section{Interrogations et examens}

L'annonce publicitaire parue dans La Sucrerie Belge de $1897,{ }^{46}$ indiquait que chaque semaine une demi-journée était consacrée à la visite d'un établissement industriel sur lequel les élèves devaient fournir un rapport. Les manipulations en laboratoire étaient cotées chaque jour et les résultats des examens mensuels étaient adressés aux parents, accompagnés de l'appréciation du directeur.

Un registre appelé « Examens et interrogations mensuelles» de 1894 jusqu'à 1899 contient toutes les points des interrogations. Pendant l'année 1984-1895 il y eut des interrogations le 29 novembre 1894, le 17 janvier 1895, le 14 mars 1885, le 25 avril 1895 et le 27 juin 1895 . Pour chaque étudiant et chaque cours le directeur donnait son appréciation. 47

Dans les brochures donnant les programmes des cours de 1901 et 1908 il y avait parmi les dispositions réglementaires un article sur les examens. L'examen de sortie se faisait devant un jury qui se composait du délégué de la Société Générale des Fabricants de Sucre de Belgique, des membres du corps professoral, des membres étrangers, choisis parmi les sommités scientifiques et industrielles. L'examen final comportait trois épreuves: la première épreuve comportait deux journées de travail au laboratoire, la deuxième et troisième épreuves étaient orales. Un tiers des points était attribué au travail de l'étudiant pendant l'année, un seconde tiers correspondait au travail des deux premières épreuves de l'examen final subies devant les professeurs et le troisième tiers s'appliquait à l'examen devant le Jury étranger. Pour avoir une idée de ce jury on peut lire dans La Vallée du Geer du 25 Août 1904 que celui-ci comprenait: M.M. Henri Pellet (Paris), Emile Légier (Paris), Emile Silz (Paris), F. Verbièse (Lille), A. Aulard (Genappe), Beyaert (Maastricht), J. Delaite, H. Philippot et E. Vrancken (Liège), Schmidt (Tirlemont) et Thiry (d'Orp).

Il y avaient quatre grades possibles: avec la plus grande distinction (au moins 90\%), avec grande distinction (au moins 80\%), avec distinction (au moins $70 \%$ ), et d'une manière satisfaisante (au moins 60\%). Un registre appelé " Procès-verbaux des examens de sortie " pour les années 1905 à 1909 nous donnent les résultats de tous les examens ainsi que les examens de sorties avec les membres du jury étranger (chaque fois avec Henri Pellet de Paris comme président et Paul le Bussy comme secrétaire). ${ }^{48}$

\section{Diplômés de l'Ecole Sucrière Belge - Institut Polytechnique}

L'annuaire de l'Association Royale des Ingénieurs Techniciens sortis de l'Institut Polytechnique de la Ville de Liège de 1961-1962, reproduit les noms des anciens diplômés à partir de 1891 jusqu'au 1949 par année de promotion et avec la nationalités noms sont classés alphabétiquement. En moyenne l'âge des diplômés était de 21 ans, mais celui-ci variait entre 16 et 31 ans. 49

On peut constater qu'à partir de 1891 le nombre de diplômés va en augmentant jusqu'en 1895, où on note 20 diplômés, puis le nombre de diplômés se situe entre 10 et 20 diplômés par année (avec quelques exceptions pour 1900: 22 diplômés; 1908: 26 diplômés et en 1924: 23 diplômés). Une chose très frappante est la présence des diplômés étrangers: à partir de 1899 le nombre de diplômés étrangers dépassent le nombre de diplômés Belges. Ce nombre augmente tellement qu'il y avait des années où il n'y a qu'un diplômé Belge (1906, 1919 et 1912). Pour la période 1891-1914 sur un total de 362 diplômés (avec en moyenne 15

46 Ibidem 29.

47 Ibidem 24.

48 Ibidem 24.

49 Ibidem 29. 
Hendrik Deelstra et Michel Peters

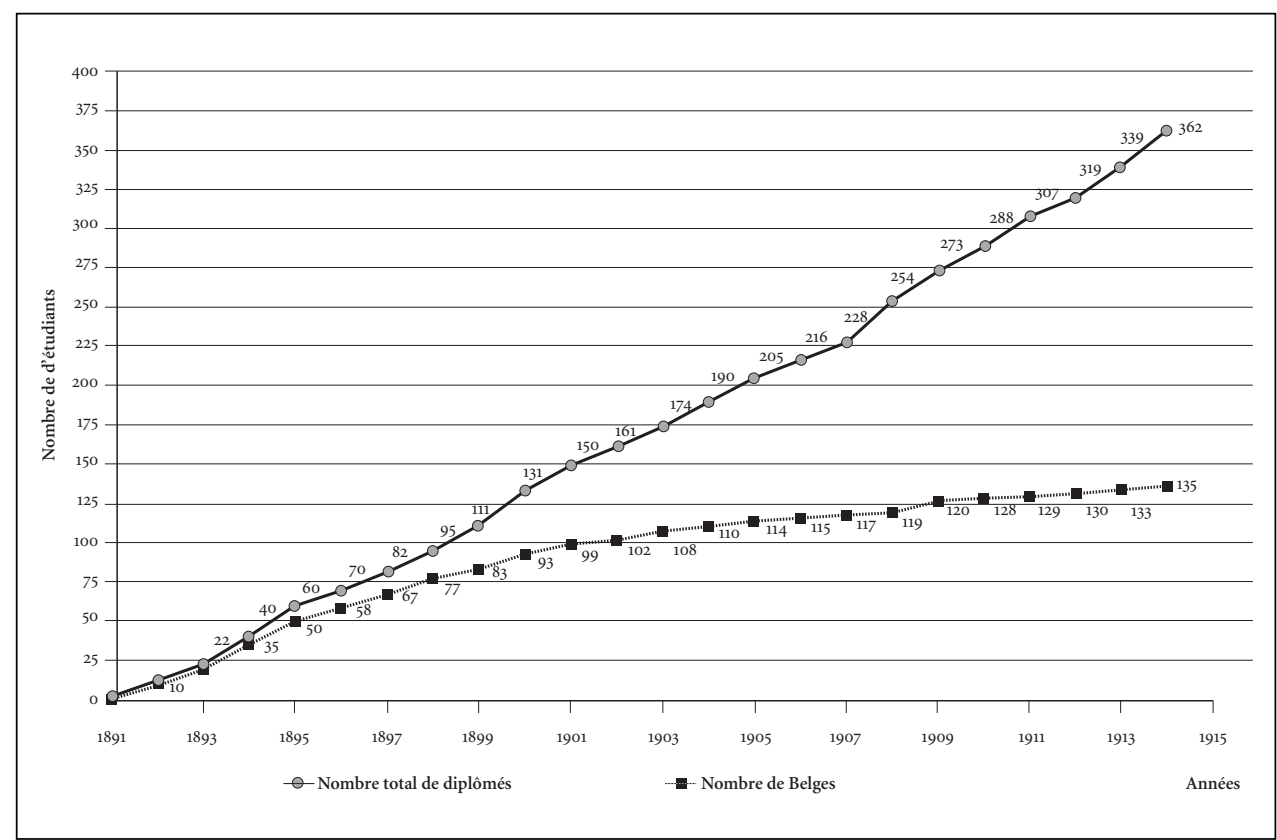

Nombre total de diplômés de L'cinstitut Polytechnique de Glons - Liège entre 1891 et 1914

diplômés par année) on compte seulement 134 Belges ( $\pm 37 \%$ ). Par contre il y avait 63 diplômés Polonais, 61 diplômés Italiens, 24 diplômés Français et 22 diplômés Espagnols. ${ }^{50}$ A partir de 1899 il y avait chaque année plus de diplômés étrangers que Belges; on peut presque dire que le nombre de diplômés Belges entre 1910 et 1914 est négligeable.

Ces données montrent clairement comment l'Ecole Sucrière de Glons était attractive pour les étrangers, non seulement des pays Européens, mais aussi de l'Egypte (trois diplômés), du Nicaragua, du Brésil, et d'autres pays. Pourquoi cette attraction? Rien ne nous le renseigne. Le fait d'avoir trouvé deux programmes de cours (de 1901 et 1908) dans la Bibliothèque des Affaires Etrangères nous donne une indication sur le fait que certainement les Ambassades Belges à l'étranger jouaient un rôle non négligeable dans la publicité faite pour cette école. Les noms et les nationalités ne nous fournissent aucune donnée quant à la qualité des diplômés. L'annuaire de 1961-1962 se dit «forcement incomplet, parce que l'occupation de l'Institut de Glons en 1914 par les troupes Allemandes avait provoqué l'incendie des archives ».

Heureusement la recherche dans les archives du journal hebdomadaire La Vallée $d u$ Geer a fourni pour quatre promotions plus de détails: ils s'agit des années 1904, 1905, 1906 et $1908 .{ }^{51}$ Plusieurs étudiants logeaient chez des habitants de Glons. Ils provenaient tous ou presque de familles aisées. Les résultats publiés dans La Vallée du Geer donnent les noms exacts et en plus les lieux de naissance. A titre d'exemple, citons quelques noms

50 Quoique la Pologne n'exista plus à partir de 1795, vers les années 1900 la partie russe de la Pologne avait obtenu une certaine autonomie en tant que province russe. On parlait alors de Pologne russe.

51 La Vallée du Geer, ${ }^{\circ} 35$ du 25 août 1904; idem n ${ }^{\circ} 34$ du 24 août 1905; idem nº 34 du 23 août 19o6; idem nº 35 du 27 août 1908. 


\section{L'Ecole Sucrière Belge de Glons}

évocateurs: de Féligonde, de Frabel, Chevalier de Spirlet, Félix de Meauxsoone.... La Vallée $d u$ Geer du 25 août 1904 énumérait les diplômés de l'année (1903-1904). Sur 30 élèves inscrits, 23 ont été examinés et 16 ont obtenu le diplôme d'ingénieur sucrier, indique la gazette qui cite ceux qui ont obtenu une grande distinction:

Enrico Volta de Sabbioneta, Italie

Zygmunt Trzcinski de Rydzeurie, Pologne

Achille Dudur de Courcelles, Hainaut

Charles de Féligonde d'Ebreuil Allier, France

D'autres ont décroché des distinctions:

Georges Warzée d'Iglesias, Sardaigne

Umberto Violini de Padoue, Italie

Ricardo Argos de Carthagène, Espagne

Jacques Mertens de Melitopol (Crimée), Russie.

Il faut reconnaître que peu de belges, curieusement, figuraient au palmarès. Les résultats mentionnés dans les extraits de La Vallée du Geer et les résultats trouvés dans le Bulletin Technique ${ }^{2}$ organe de l'Institut Polytechnique de Glons pour l'année 1910 sont résumés dans le Tableau: cet aperçu des lauréats et de leur grade pour les cinq années montrent que sur un total de 85 diplômés il y avaient 46 lauréats avec un grade de distinction ou plus. Parmi ces 46 diplômés on ne compte que deux belges!

Les diplômés de l'Ecole Sucrière Belge, après l'Institut Polytechnique trouvaient facilement du travail dans l'industrie sucrière. Le programme de cours de 1927 donne comme référence pour cent diplômés la situation occupée dans l'industrie. Parmi ces cent anciens élèves on trouve encore 15 personnes avec le diplôme d'ingénieur sucrier, parmi lesquels trois diplômés de l'étranger (donc diplômés avant 1906) (un Arménien et deux Italiens). La plus grande majorité de ces personnes occupaient une fonction de directeur de sucrerie soit en Belgique soit à l'étranger.

\section{La vie étudiante}

Quelques données sur la vie estudiantine, avec tant de nationalités, sont trouvées dans $\mathrm{La}$ Vallée du Geer, dans l'annuaire 1961-1962 et dans la revue mensuelle Le Bulletin Technique. La Vallée du Geer du 26 mai 1904 contient un article concernant une manifestation de sympathie envers Honoré Duvivier, répétiteur à l'occasion de sa fête patronale St. Honoré. M. Lefèvre de Roccour prenait la parole. Il louait Honoré Duvivier pour le zèle et son dévouement à initier les étudiants aux travaux du Laboratoire de Chimie. Il avait remis un souvenir au héros de la fête et terminait son discours en s'adressant aux étudiants étrangers en leur disant: " qu'ils étaient étranger à cette terre, mais qu'ils ne l'étaient pas dans nos cœurs ». L'année suivante, le journal rapporte le 23 mai 1905 à l'occasion d'une fête analogue. M. Lefebvre de Roccour traduisait les sentiments de ces camarades par un discours.

52 Le Bulletin Technique, Revue mensuelle illustrée. Organe de l'Institut Polytechnique de Glons et de l'Association des élèves et anciens élèves de cet Institut. Rédacteur en chef: E. Coffineau, 1909-1911, Publications de l'Institut. 


\section{Hendrik Deelstra et Michel Peters}

L'annuaire 1961-1962 mentionne l'histoire de l'Association Royale des Ingénieurs Techniciens de l'Institut Polytechnique de la ville de Liège. Selon l'historique, le 8 avril 1891 déjà des étudiants auraient décidés de se grouper en une association avec comme but de maintenir leurs relations après la sortie de l'Institut. Cependant le premier tome du Bulletin Technique de 1909-1910 mentionne que cette association a été fondée à Glons le 19 janvier 1909. Dans son discours de l'inauguration de cette association Paul le Bussy ne renvoya pas à ce fait datant de 1891 !

Le Bulletin Technique, dont on a trouvé que deux tomes (155 pages) contient beaucoup d'information sur la vie étudiante à Glons: des excursions (Tongres, Anvers, Ougrée, etc.), l'inauguration d'un local. Les procès verbaux des réunions et les sujets traités donne une idée de la vie estudiantine: des discussions sur les statuts (les jeux de hasard étaient absolument défendus dans le local de l'association), la casquette d'étudiant et les couleurs pour le liseré (ces couleurs devaient être neutres), des questions sur l'interrogation d'un certain professeur, etcetera.

\section{Conclusions}

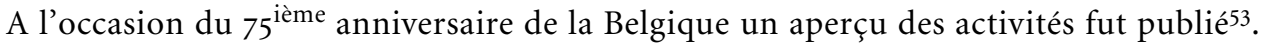
L'auteur mentionnait qu'on 1905 sous l'enseignement agricole il y avait outre l'école de Glons, une deuxième école sucrière établie à Waremme. En effet, le 15 février 1895 fut fondée au Collège Saint-Louis une école de sucrerie. ${ }^{54}$ Cette école connut un grand succès national et international. Des registres des diplômés on peut relever que pendant les 33 ans de vie de cette section il y eut 360 étudiants (dont $38 \%$ étrangers). Si on compare les diplômés pour Glons pour la même période on peut compter 551 diplômés (dont 52\% étrangers). Quelques registres pour Waremme nous montrent que les étudiants étrangers - comme pour Glons - obtenaient les meilleurs résultats.

Le 29 juillet 1905 le conseil communal de la ville de Saint-Ghislain approuva le programme d'une nouvelle école professionnelle de sucrerie. ${ }^{55}$ Cette école subira beaucoup de modifications. Le nombre de diplômés ingénieurs chimistes est beaucoup moins important qu’à Glons et Waremme. Il est cependant impressionnant de noter que l'école de Glons eut un tel succès à l'étranger. Pendant la période de 1900 à 1914, il y eut 251 diplômés, dont 80\% d'étrangers. Ce résultat est certainement dû au niveau du corps professoral et aux membres du jury étranger avec comme président le très célèbre spécialiste du sucre Henri Pellet. ${ }^{56}$

53 La Belgique 1830-1905 (Bruxelles 1905) 222-223.

54 André Loumaye, 'Histoire du Collège Saint Louis à Waremme (1855-1969)', Cahiers Waremmiens, n 26, août 1997, Ed. M. Joachim, commission d'histoire et de littérature de la ville de Waremme, 39-40.

55 G. Hénaut, 'L'Institut de Chimie de Saint-Ghislain (1905-1980),' Ann. Cercle Hist. et d'Archéologie de SaintGhislain 3 (1982) 563-574.

56 Les auteurs de cet article ont longtemps cherché une notice biographique ou une nécrologie concernant Henri Pellet, c'est finalement en contactant les Archives nationales françaises (site de Paris), détenant au travers de la base de données Léonore les dossiers des titulaires de l'Ordre de la Légion d'Honneur depuis la création de l'Ordre (et décédés avant 1954), qu'ils ont pu obtenir des documents donnant quelques aspects de la vie d'Henri Pellet et prouvant qu'il était à la fin du $19^{\text {ème }}$ siècle un des chimistes sucriers les plus importants d'Europe. Jean-Baptiste Henri Pellet dit Henri est né le 23 juillet 1848 à Saint Léger du Bois en Saône et Loire et est décédé à son domicile 148 Boulevard Magenta à Paris le 31 janvier 1918. C'est assurément le conflit mondial qui fit que sa mort passa assez inaperçue et qu'elle ne fut pas retranscrite dans La Sucrerie Belge ou le Journal des fabricants de Sucre. Henri Pellet eu une vie bien remplie et les titres dont le faire part de décès font mention montrent à quel point il avait marqué sa profession. Il servit pendant la guerre Franco-Prussienne de 1870 où il fut blessé. Pellet fut Chimiste Conseil de la Société des Sucreries et Raffinerie Say et de la Société des Sucreries et Raffinerie d'Egypte, Administrateur de la Société de Chimie Industrielle 


\section{L'Ecole Sucrière Belge de Glons}

de France, Président du Syndicat des Chimistes et Essayeurs de France, Vice-Président de l'Association des Chimistes de Sucreries et Distilleries. Il fut décoré de la Médaille militaire pour ses faits d'armes lors du conflit de 1870. Il fut fait, en France, Officier du Mérite Agricole et Chevalier de la Légion d'Honneur et en Belgique, Commandeur de l'Ordre de Léopold.

En 1881 La Sucrerie Belge 10 (1881) 433-434, reprenait un extrait du Journal des Fabricants (Français) de Sucre intitulé 'Le laboratoire de chimie de M. Henri Pellet'. On y apprend que Henri Pellet avait installé à Paris, 5, rue Fénélon, un laboratoire scientifique, industriel et agricole. Selon l'extrait, Pellet était chimisteconseil de divers établissements industriels, secrétaire général de la Société des industries chimiques et agricoles, grand prix agronomique de la Société des agriculteurs de France et lauréat de la Société industrielle du Nord. Il était considéré comme étant " assurément l'un des chimistes qui faisait le plus d'honneur à la sucrerie française ». L'extrait rappelait « la multitude de questions se rattachant à la fabrication du sucre, auxquelles M. H. Pellet avait consacré ses recherches ainsi que la part de celui-ci dans l'établissement du contrôle chimique du sucre ». L'extrait mentionnait que le but de la création du laboratoire était d'enseigner aux élèves les vrais principes de l'industrie sucrière. Enfin, cet extrait soulignait que le laboratoire était parfaitement équipé, comprenait une bibliothèque complète et que de plus il réunissait toutes les conditions pour exécuter avec précision et rapidité les analyses les plus variées ». Le journal La Sucrerie Belge a publié des notes de voyages d'Henri Pellet en Allemagne, en Autriche et en Belgique (voir La Sucrerie Belge 12 (1883) $128,146,166$ et 208$)$.

Il faut également souligner qu'Henri Pellet joua un rôle extrêmement important dans les activités de la section sucrière de l'Association Belge des Chimistes fondée en 1887. Dès la création de cette section, Pellet se montra l'un des membres les plus actifs (voir Deelstra et Fuks - note 3). Pellet à ce moment chimiste en chef des sucreries de Wanze, à Statte, par Huy est cité dans les diverses listes de membres de l'Association depuis le début jusqu'a son retour en France en 1893. Dès la première assemblée générale de l'Association, le 4 août 1887 il est élu en tant que membre du Conseil. A l'assemblée générale du 3 mai 1893, Pellet déclarait « qu'il regrettait ne plus pouvoir accepter un renouvellement de son mandat, attendu qu'il allait quitter la Belgique pour rentrer en France " (voir Bulletin de l'Association Belge des Chimistes 7 (1893) 7). A partir de ce moment et jusqu'à la réorganisation de l'Association en 1898, Pellet devint membre effectif et honoraire déclaré habitant en France.

Dans la section sucrière Pellet participa à toutes les discussions et à tous les travaux sur l'analyse du sucre de betterave. En effet, c'est sa méthode pour l'extraction du sucre de betterave qui fut choisie comme méthode standard par les chimistes sucriers (Bulletin de l'Association Belge des Chimistes 2 (1889) 97-106). Il publia dans le Bulletin 31 articles en relation avec la section sucrière et 8 articles sur les engrais proposé à la section de chimie agricole. Dans un dernier article magistral intitulé 'Les analyses de betteraves en Belgique pendant la campagne 1892-93', (voir Bulletin de l'Association Belge des Chimistes 7 (1893) 26-60), il résumait toutes les conditions nécessaires pour exécuter correctement l'analyse du sucre dans la betterave. Au Grand Concours International des Sciences et de l'Industrie de Bruxelles (1888), il obtint trois prix (deux prix de progrès et un prix d'excellence) pour ses mémoires relatifs aux questions des différents concours (Bulletin de l'Association Belge des Chimistes 4 (1890) 44-46). Entre temps il publia plusieurs livres e.a. avec son collègue français L. Biard Le Traité d'analyse chimique du sucre (voir Bulletin de l'Association Belge des Chimistes IV (1890) 225-226).

En France Pellet continua à publier e.a. 'Dosage du sucre cristallisable dans la betterave', dans Les Annales de la Science agronomique française et étrangère I (1893) 160 et Les pertes indéterminés en sucrerie (Compiègne 1895) 60). Ce mémoire fut introduit suite à un concours organisé par l'Association des Chimistes de Sucrerie et de Distillerie. Pellet fut récompensé par un prix et le mémoire fut aussi imprimé dans le Bulletin des Chimistes, sept-oct, 1895 .

Après son départ pour la France Pellet continua à avoir de très bons contacts avec ces collègues belges. Dès la fondation de la Société Technique et Chimique de Sucrerie en 1896, il fut membre (voir la Sucrerie Belge 25 (1896) 383). Sur la liste de membres pour 1903-1904, Pellet était inscrit comme membre correspondant et mentionné comme chimiste, 'Chevalier de la Légion d'Honneur'. Sur la liste de membres pour 1910-1911, Pellet était devenu membre d'honneur avec deux adresses: une à Paris l'autre au Caire en Egypte.

Terminons cette note en soulignant le travail d'expert fourni par Henri Pellet à l'étranger. Dans un article de Michel Dumoulin intitulé 'Les premières années de la présence Belge en Perse (1887-1895)', (Les archives de la revue belge d'histoire contemporaine XIII (1977) 52) on peut lire à la page 36, qu'en février 1892 la Société pour la fabrication du Sucre en Perse (soutenue par le Roi Léopold II) délégua à Téhéran un certain Henri Pellet, pour y mener une enquête d'installation des industries (e.a. du sucre). Pellet expédia en Belgique des échantillons de betteraves qui furent analysés dans les laboratoires de la Raffinerie Tirlemontoise, ces analyses concluaient favorablement en faveur des chances de succès d'une sucrerie en Perse.

Habitant Paris, Pellet fut approché en 1902 par le Belge Henri Naus (né à Hasselt en 1875 et décédé à Bruxelles en 1938) qui lui demanda de l'aider en Egypte car il avait des problèmes de raffinage de sucre de canne (voir le mémoire de U.M. Kupferschmidt 'Henri Naus Bey: Retrieving the Biography of a Belgian Industrialist in Egypt', Koninklijke Academie voor Overzeese Wetenschappen. Klasse Morele en Politieke Wetenschappen, nieuwe reeks, boek 52, afl. 2, (Brussel 1999) 44). L'auteur écrit: « with great perseverance, and help 


\section{Hendrik Deelstra et Michel Peters}

\section{SUMMARY}

\section{The Belgian sugar school of Glons}

This article deals with the start in 1889 and the evolution till 1914 of the first Sugar technical school in Belgium located in Glons-Liège. In the literature on the history of sciences in Belgium this school has been unjustly neglected or forgotten. This is probably due to the total destruction of the archives in 1917 by the German occupiers.

The history of the creation of a School for sugar chemists and technicians goes back to the early 1880's, when the need was realised for the creation of such a High Technical School, to support and improve the most important agro-industrial industry in Belgium: the beet-sugar production. However there was at that time no consensus how to create and organize such an Institute.

In 1889 , E. Nihoul started in Glons a private sugar technical school for the direct training of specialists for all functions in the sugar industry, especially that of sugar chemists. This article describes the history of this school from 1889 till 1914. The school rapidly gained a great reputation and became extremely attractive for students outside Belgium. During the period 1900 till 1914 the school produced 251 qualified specialists of which $80 \%$ were foreigners. The school moved to Liège in 1917 and after 1932 involved in fusions with different Technical High Schools to form the now well-known 'Hautes Ecoles Rennequin Sualem' in Liège.

of the most prominent and innovating sugar expert of the time, named $\mathrm{H}$. Pellet, he developed a technique to overcome the difficulties in the refining process " (voir aussi la nécrologie de H. Naus, Bulletin de l'Association des chimistes (français) 55 (1938) 961, où il est mentionné que H. Naus devint directeur de la grande Sucrerie Egyptienne de Nag-Hamadi en 1902 avec le concours de Henri Pellet.

Enfin il faut souligner que Henri Pellet fit partie du premier Congrès International de Chimie appliquée à Anvers-Bruxelles en 1894 et du premier Congrès International Technique et Chimique de Sucrerie et de Distillerie, tenu à Liège du 5 au 10 juin 1905. (Congrès qui faisait concurrence au Congrès de Chimie et de Pharmacie, tenu également à Liège du 27 à 30 juillet 1905). Au banquet de ce premier congrès à Liège, Henri Pellet porta un toast à la santé des fabricants de sucre de betteraves et de sucre de cannes. Il débuta son discours en soulignant qu'il était bien au courant des activités de ces deux secteurs, qui se faisaient souvent de la concurrence. Il y détaille également une vue de l'ensemble des différents pays où il existait des cultures de la canne et d'extraction de son sucre (voir la Sucrerie Belge 33 (1905) 537-539). 